\title{
Simulating Quantum Fields with Cavity QED
}

\author{
Sean Barrett, ${ }^{1, *}$ Klemens Hammerer, ${ }^{2,3}$ Sarah Harrison, ${ }^{4,2}$ Tracy E. Northup, ${ }^{5}$ and Tobias J. Osborne ${ }^{2}$ \\ ${ }^{1}$ QOLS, Blackett Laboratory, Imperial College London, London SW7 2BW, United Kingdom \\ ${ }^{2}$ Institute for Theoretical Physics, Leibniz University, 30167 Hannover, Germany \\ ${ }^{3}$ Institute for Gravitational Physics, Leibniz University, 30167 Hannover, Germany \\ ${ }^{4}$ Department of Mathematics, Royal Holloway, University of London, Egham TW2O OEX, United Kingdom \\ ${ }^{5}$ Institut für Experimentalphysik, Universität Innsbruck, 6020 Innsbruck, Austria \\ (Received 8 November 2012; revised manuscript received 25 January 2013; published 26 February 2013)
}

\begin{abstract}
As the realization of a fully operational quantum computer remains distant, quantum simulation, whereby one quantum system is engineered to simulate another, becomes a key goal of great practical importance. Here we report on a variational method exploiting the natural physics of cavity QED architectures to simulate strongly interacting quantum fields. Our scheme is broadly applicable to any architecture involving tunable and strongly nonlinear interactions with light; as an example, we demonstrate that existing cavity devices could simulate models of strongly interacting bosons. The scheme can be extended to simulate systems of entangled multicomponent fields, beyond the reach of existing classical simulation methods.
\end{abstract}

DOI: 10.1103/PhysRevLett.110.090501

Modelling interacting many-particle systems classically is a challenging yet tractable problem. However, in the quantum regime, it becomes rapidly intractable, owing to the dramatic increase in the number of variables required to describe the system. Feynman [1] realized that an alternate approach would be to exploit quantum mechanics to carry out simulations beyond the reach of classical computers. This idea was the basis of Lloyd's simulation algorithm [2], a procedure for a digital quantum computer to simulate the dynamics of a strongly interacting quantum system. In contrast, there is also an analogue approach to quantum simulation, where the simulator's Hamiltonian is tailored to match that of the simulated system [3]. The complementary aspects of the analogue and digital methods, reviewed in Refs. [3-7], have led to a host of recent experiments [8-16].

To date, most experimental implementations of quantum simulation algorithms have been focussed on the task of simulating quantum lattice systems, with comparatively less attention paid to systems with continuous degrees of freedom. The archetypal example of a quantum system with a continuous degree of freedom is the quantum field. Currently, quantum simulations of quantum field theories have relied on discretization of the dynamical degrees of freedom. One body of recent theoretical work is focussed on the analogue simulation of discretized quantum fields, using cold atoms in optical lattices [17-21] and coupled cavity arrays [22-24]. Complementing this are proposals for digital quantum simulation on a universal quantum computer of the zero-temperature [25] and thermal [26] dynamics of non-Abelian gauge theories and, more recently, a digital quantum simulation $[27,28]$ of scattering processes of a discretized $\lambda \phi^{4}$ quantum field.

In this Letter, we report on an analogue algorithm to simulate the ground-state physics of a one-dimensional
PACS numbers: 03.67.Ac, 03.70.+k, 42.50.Ex, 42.50.Pq

strongly interacting quantum field using the continuous output of a cavity-QED apparatus [29-33]. Our method involves no discretization of the dynamical degrees of freedom; the simulation register is the continuous electromagnetic output mode of the cavity. The variational wave function generated in this way, therefore, belongs to an extremely expressive class, namely the class of continuous matrix product states, as we will show. We argue that our approach is already realizable with state-of-the-art cavityQED technology.

We concentrate on simulating quantum fields modelling collections of strongly interacting bosons in one spatial dimension. These systems are compactly described in second quantization using the quantum field annihilation and creation operators $\hat{\psi}(x)$ and $\hat{\psi}^{\dagger}(x)$, which obey the canonical commutation relations $\left[\hat{\psi}(x), \hat{\psi}^{\dagger}(y)\right]=\delta(x-y)$. The task is to determine the ground state of a given fieldtheoretic Hamiltonian $\hat{\mathcal{H}}\left(\hat{\psi}, \hat{\psi}^{\dagger}\right)$. The prototypical form of such a Hamiltonian is

$$
\hat{\mathcal{H}}=\int(\hat{T}+\hat{W}+\hat{N}) d x
$$

where $\quad \hat{T}=\frac{d \hat{\psi}^{\dagger}(x)}{d x} \frac{d \hat{\psi}(x)}{d x}, \quad \hat{W}=\int w(x-y) \hat{\psi}^{\dagger}(x) \hat{\psi}^{\dagger}(y)$ $\hat{\psi}(y) \hat{\psi}(x) d y$, and $\hat{N}=-\mu \hat{\psi}^{\dagger}(x) \hat{\psi}(x)$ describe the kinetic energy, two-particle interactions with potential $w(x-y)$, and the chemical potential, respectively. Our approach provides a quantum variational algorithm for finding the ground states of an arbitrary Hamiltonian that is translation-invariant and consists of finite sums of polynomials of creation/annihilation operators and their derivatives.

The apparatus proposed to simulate the ground-state physics of $\hat{\mathcal{H}}$ is a single-mode cavity coupled to the quantum degrees of freedom of some intracavity medium (Fig. 1); our proposal is not tied to the specific nature of 


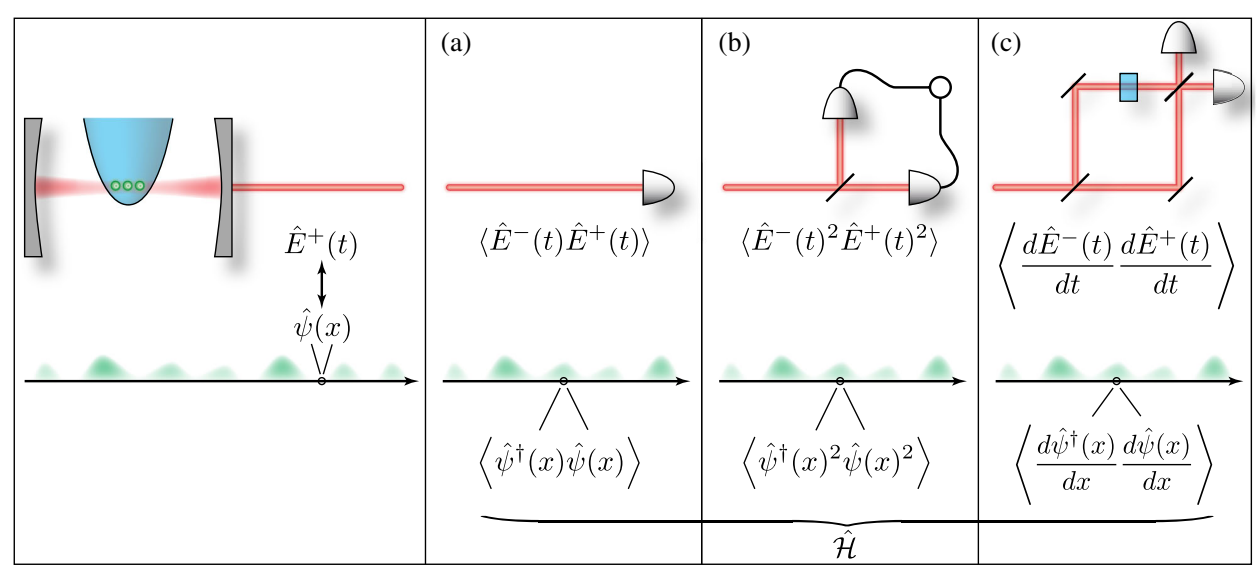

FIG. 1 (color online). The output field $\hat{E}^{+}(t)$ of a cavity-QED system is identified with a bosonic quantum field $\hat{\psi}(x)$. Since optical detection schemes correspond to expectation values of quantum-field operators, $\langle\hat{\mathcal{H}}\rangle$ can be estimated via independent measurements of the cavity field. For example, the operators $\hat{N}, \hat{W}$, and $\hat{T}$ of Eq. (1) are determined, respectively, from measurements of (a) the output-field intensity, (b) Hanbury Brown-Twiss correlations, and (c) an interferometer with variable path length.

the medium, so long as one or more tunable nonlinear interactions are present that are sufficiently strong at the single-photon level. Below we consider the example of a single trapped atom coupled to the cavity via electronic transitions. The system is described by a Hamiltonian $\hat{H}_{\text {sys }}(\lambda)$ that depends on a set of controllable parameters $\lambda$, for example, externally applied fields. When the cavity is driven, either directly through one of its mirrors or indirectly through the medium, the intracavity field relaxes to a stationary state, and the cavity emits a steady-state beam of photons in a well-defined mode.

The crucial idea underlying our proposal is to regard the steady-state cavity output as a continuous register recording a variational quantum state $|\Psi(\lambda)\rangle$ of a onedimensional quantum field with control parameters $\lambda$ as the variational parameters. This representation is chosen so that the spatial location $x$ of the simulated translationinvariant field is identified with the value of the timestationary cavity output mode exiting the cavity at time $t=x / s$. The arbitrary scaling parameter $s$ is included in the set of variational parameters $\lambda$. We complete this identification by equating the annihilation operator $\hat{\psi}(x)$ of the simulated quantum field with the field operator $\hat{E}^{+}(t)$ for the positive-frequency electric field of the cavity output mode [34], via $\hat{\psi}(x)=\hat{E}^{+}(t) / \sqrt{s}$.

Recall that the variational method proceeds by minimizing the average energy density of the variational state $f(\lambda)=\langle\Psi(\lambda)|\hat{T}+\hat{W}+\hat{N}| \Psi(\lambda)\rangle$ over the variational parameters $\lambda$. A key point in our scheme is that, with the identification of the field operators $\hat{E}^{+}(t)$ and $\hat{\psi}(x)$ in hand, the value of $f(\lambda)$ can be determined from standard optical measurements on the cavity output field, namely the measurement of Glauber correlation functions [35,36], see Fig. 1. This result is easily seen for the Hamiltonian of Eq. (1). Thanks to the linearity of the expectation value, we can separately measure $\langle\hat{T}\rangle,\langle\hat{W}\rangle$, and $\langle\hat{N}\rangle$. The expectation value of the chemical potential term corresponds to a function of the intensity of the output beam via $\langle\hat{N}\rangle=$ $-\frac{\mu}{s}\left\langle\hat{E}^{-}(t) \hat{E}^{+}(t)\right\rangle$. The kinetic energy term $\langle\hat{T}\rangle$ corresponds to the limit

$$
\begin{aligned}
\langle\hat{T}\rangle= & \lim _{\epsilon_{1}, \epsilon_{2} \rightarrow 0} \frac{1}{s^{3} \epsilon_{1} \epsilon_{2}}\left[g^{(1)}\left(t+\epsilon_{1}, t+\epsilon_{2}\right)-g^{(1)}\left(t+\epsilon_{1}, t\right)\right. \\
& \left.-g^{(1)}\left(t, t+\epsilon_{2}\right)+g^{(1)}(t, t)\right],
\end{aligned}
$$

where $g^{(1)}\left(t_{1}, t_{2}\right)=\left\langle\hat{E}^{-}\left(t_{1}\right) \hat{E}^{+}\left(t_{2}\right)\right\rangle$; this quantity can be estimated by choosing a finite but small value for $\epsilon_{1}$ and $\epsilon_{2}$. Note that this procedure does not amount to a simple space discretization because the output is a continuous quantum register. The final term $\langle\hat{W}\rangle$ depends on two-point spatial correlation functions $\left\langle\hat{\psi}^{\dagger}(x) \hat{\psi}^{\dagger}(y) \hat{\psi}(y) \hat{\psi}(x)\right\rangle$, which translate to measurements of $g^{(2)}\left(t_{1}, t_{2}\right)=$ $\left\langle\hat{E}^{-}\left(t_{1}\right) \hat{E}^{-}\left(t_{2}\right) \hat{E}^{+}\left(t_{2}\right) \hat{E}^{+}\left(t_{1}\right)\right\rangle$. The detection schemes to estimate all terms in the showcase Hamiltonian (1) are presented in Fig. 1. From a wider perspective, any Glauber correlation function $g^{(n, m)}=\left\langle\hat{E}^{-}\left(t_{1}\right) \cdots \hat{E}^{-}\right.$ $\left.\left(t_{n}\right) \hat{E}^{+}\left(t_{m}^{\prime}\right) \cdots \hat{E}^{+}\left(t_{1}^{\prime}\right)\right\rangle$, i.e., any $n+m$ point field correlation function composed of $n$ creation operators $E^{-}$and $m$ annihilation operators $E^{+}[35,36]$, can be measured with similar, albeit more complex, setups, such as in Refs. [37,38]. Thus, upon identifying $E^{-}(t)$ and $E^{+}(t)$ with $\psi^{\dagger}(x)$ and $\psi(x)$, respectively, our scheme admits the measurement of any equivalent energy density $\propto\left\langle\hat{\psi}^{\dagger}\left(x_{1}\right) \cdots \hat{\psi}^{\dagger}\left(x_{n}\right) \hat{\psi}\left(x_{m}^{\prime}\right) \cdots \hat{\psi}\left(x_{1}^{\prime}\right)\right\rangle$, and, therefore, ultimately the simulation of arbitrary Hamiltonians $\hat{\mathcal{H}}\left(\hat{\psi}, \hat{\psi}^{\dagger}\right)$.

Once $f(\lambda)=\langle\Psi(\lambda)|\hat{\mathcal{H}}| \Psi(\lambda)\rangle$ has been experimentally estimated for a given $\lambda$, the next step is to apply the variational method to minimize $f(\lambda)$. Minimization is carried out by adaptively tuning the parameters $\lambda$ in the system Hamiltonian $\hat{H}_{\text {sys }}(\lambda)$ and iteratively reducing $f(\lambda)$, 
for example, using a standard numerical gradient-descent method. Once the optimum choice of $\lambda$ is found, the resulting cavity output field is a variational approximation to the ground state of $\hat{\mathcal{H}}$, and relevant observables of the field theory can be directly measured using the detection schemes of Fig. 1. We emphasize that our method applies also to cases where a numerical estimation of $f(\lambda)$ cannot be performed efficiently due to the size and complexity of the system to be simulated, and we suggest that this is exactly the strength of our approach. Moreover, the optimization may be performed experimentally without theoretically calculating the cavity-QED system dynamics; indeed, it is not necessary to accurately characterize $H_{\text {sys }}$ or its relation to the adjustable parameters $\lambda$.

Why should the stationary output of a cavity-QED apparatus be an expressive class capable of capturing the ground-state physics of strongly interacting fields? It is possible to show [39] that such states are of a continuous matrix product state (CMPS) type, a variational class of quantum field states recently introduced for the classical simulation of both nonrelativistic and relativistic quantum fields [40-43]. These states are a generalization of matrix product states [44-47], which have enjoyed unparalleled success in the study of strongly correlated phenomena in one dimension in conjunction with the density matrix renormalization group $[48,49]$. It turns out that all quantum field states admit a CMPS description, providing a compelling argument for their utility as a variational class [50]. Crucially, the CMPS formalism turns out to be identical to the input-output formalism of cavity QED [51]. This identification was anticipated in Refs. [40,41,52,53], and we elucidate it further in Ref. [39]. It implies that quantum field states emerging from a cavity are of the CMPS type and thus fulfill the necessary conditions for being a suitable and expressive class of variational quantum states.

Even though cavity-QED output states are of the CMPS type, can a realistic system in the presence of decoherence reproduce the relevant physics of a strongly interacting quantum field? As a test case, we demonstrate that the paradigmatic cavity-QED system, comprising a single trapped atom coupled to a high-finesse cavity mode, is capable of simulating the ground-state physics of an equally paradigmatic field, namely, the Lieb-Liniger model [54]. This model describes hard-core bosons with a delta-function interaction and is given by Eq. (1) with $w(x-y)=v \delta(x-y)$, where $v$ describes the interaction strength. Our simulator consists of a two-level atom interacting with one cavity mode, described (in a suitable rotating frame) by the on-resonance Jaynes-Cummings Hamiltonian

$$
\hat{H}_{\mathrm{sys}}=g\left(\hat{\sigma}^{+} \hat{a}+\hat{\sigma}^{-} \hat{a}^{\dagger}\right)+\Omega\left(\hat{\sigma}^{+}+\hat{\sigma}^{-}\right),
$$

where $\hat{\sigma}^{+}$is the atomic raising operator and $\hat{a}$ is the cavity photon annihilation operator, $g$ the atom-cavity coupling, and $\Omega$ the laser drive. The cavity-QED Hamiltonian $\hat{H}_{\text {sys }}$ can be realized in various experimental architectures [29-33]. Here we choose the example of a trapped calcium ion in an optical cavity, with which tunable photon statistics have previously been demonstrated [55], and we show in the Supplemental Material [39] how $g$ and $\Omega$ can function as variational parameters.

In an experiment, to measure the variational energy density $f(\lambda)$, the output beam would be allowed to relax to steady state, the intensity $I, g^{(1)}$, and $g^{(2)}$ functions estimated as depicted in Fig. 1, and $f(\lambda)$ finally estimated from postprocessing this data. Measurement schemes (a) and (b) of Fig. 1 are just the standard laboratory techniques of photon detection and Hanbury Brown-Twiss interferometry. Measurement scheme (c) represents an interferometer with variable path length that is used to estimate the derivative of the quantum field in the kinetic energy term $\langle\hat{T}\rangle$. Length shifts on the mm scale correspond to ps values of $\epsilon_{1}$ and $\epsilon_{2}$ in the estimation of $\langle\hat{T}\rangle$; as these values are six orders of magnitude smaller than the relevant time scales of the experiment, they are considered sufficiently small.

Obviously, the test model chosen here is simple enough to admit a classical simulation, which we carry out for the experimental parameters of Ref. [56]. Exploiting a simple gradient-descent method, we find the values of $\lambda=$ $\{g, \Omega, s\}$ that minimize $f(\lambda)$ for a given value of $v$. This procedure is repeated over a range of $v$ values of interest, and the corresponding optimized values of $\lambda$ are then used to compute quantities of interest, e.g., spatial-correlation functions as shown in Fig. 2. Remarkably, we find that just these three variational parameters $\lambda=(g, \Omega, s)$, when varied in the experimentally feasible parameter regime of Refs. [55,56] in the presence of losses, allow for a quantum simulation of Lieb-Liniger ground-state physics. One expects that the ground-state approximation would improve by increasing the dimension of the auxiliary system and by allowing sufficiently general internal couplings and couplings to the field. In the context of atom-cavity systems, this can be done by making use of the rich level structure of atoms (i.e., Zeeman splittings) and making use of motional degrees of freedom. For sufficiently complex intracavity dynamics, a classical simulation will become unfeasible, and at the same time it becomes conceivable that such a simulator will outperform the best classical methods.

The reliability of a quantum simulator can be compromised by decoherence effects, as was recently emphasized in Ref. [57]. Our simulation of the ion-cavity system includes both cavity decay (at rate $\kappa$ ) and decay of the ion due to spontaneous emission (at rate $\gamma$ ). The cavity decay rate rescales the parameter $s$ linking measurement time and simulated space, and thus it can be considered as a variational parameter itself. We emphasize that cavity decay does not function as a decoherence channel in our scheme but is rather an essential element of the CMPS formalism. In contrast, spontaneous emission sets the limit 




FIG. 2 (color online). Two-particle correlations in the LiebLiniger model are reproduced in simulations of an ion-trap cavity experiment. This critical model exhibits a transition between the superfluid regime for $v \approx 0$ and the Tonks-Girardeau regime for $v \gg 0$, which is seen in the value of the correlation function at $t=0$. (a) The Lieb-Liniger ground state is simulated for interaction strengths $v=\{0.07$ (red dotted), 3.95 (orange dashed), 60.20 (blue dotdashed), 625.95 (green dotdotdashed)\}, and correlation functions $\left\langle\hat{\psi}^{\dagger}(0) \hat{\psi}^{\dagger}(x) \hat{\psi}(x) \hat{\psi}(0)\right\rangle$ calculated as in Refs. [40,41] using 338 variational parameters. (b) Two-photon correlation functions $g^{(2)}(t)$ for an experiment with the parameters of Ref. [56]. Although there are visible differences, with just three variational parameters $\{g, \Omega, s\}$ the transition in the correlation functions is approximately reproduced. It is worth emphasizing how unusual it is for a variational calculation with only a few parameters to reproduce anything more than the coarsest features of a correlation function, e.g., if mean-field theory is used one does not obtain nontrivial correlators. Strikingly, the transition in (a) is captured even in the presence of realistic decay channels (inset). Note that this transition is analogous to that observed in Ref. [55].

for the timescale over which coherent dynamics can be observed. For our present example, we can show that the regime of strong cooperativity $\mathcal{C}=g^{2} / \kappa \gamma \gtrsim 1$ is sufficient to allow a simulation of the Lieb-Liniger model despite detrimental losses. The Lieb-Liniger model exhibits nontrivial variations (Friedel oscillations) of the two-point correlation function on a length scale $\xi=$ $\left\langle\hat{\psi}(x) \hat{\psi}^{\dagger}(x)\right\rangle^{-1}$. This length scale in the simulated model translates to a time scale over which the stationary output field (as the simulating register) should exhibit similar nontrivial features. From the previously established scaling rule one finds that the required time scale is $\tau=\xi / s=$ $\left\langle\hat{E}^{-}(t) \hat{E}^{+}(t)\right\rangle^{-1}$. By means of the cavity input-output relations, we relate the output photon flux to the mean intracavity photon number $\left\langle\hat{E}^{-}(t) \hat{E}^{+}(t)\right\rangle=\kappa\left\langle\hat{a}^{\dagger} \hat{a}\right\rangle$. In the bad cavity limit $\kappa \gg g$ the cavity mode can be adiabatically eliminated. In this case $\left\langle a^{\dagger} a\right\rangle \simeq(g / \kappa)^{2}$, such that $\tau=\kappa / g^{2}$. On the other hand, the characteristic decoherence time of the ion is determined by the spontaneous emission rate $2 \gamma$. Beyond a time $1 / 2 \gamma$, the second order correlation function $g^{(2)}$ will be trivial. We, therefore, demand $\tau \lesssim 1 / 2 \gamma$, which is equivalent to the requirement of a large cooperativity $\mathcal{C} \geq 1$. For the exemplary case of the ion-cavity system considered above, the cooperativity was indeed $\mathcal{C} \simeq 1.8$, see Supplemental Material [39]. While equivalent conditions must be determined on a case-bycase basis, we expect that nontrivial quantum simulations in cavity QED will not be possible in the weak-coupling regime. Finally, there are overall losses associated with scattering and absorption in cavity mirrors, detection path optics, and photon counter efficiency. However, while these losses reduce the efficiency with which photon correlations are detected, they do not otherwise affect the system dynamics.

A natural question is when our scheme would provide a practical advantage over classical computers in the simulation of quantum fields. We expect this to be the case in particular for the simulation of fields with multiple components, or species of particles, with canonical fieldannihilation operators $\hat{\psi}_{\alpha}(x), \alpha=1,2, \ldots, N$. This situation arises in at least two settings: first, for vector bosons in gauge theories with gauge group $S U(N)$, and second, in the nonrelativistic setting of cold atomic gases with multiple species. Variational calculations using CMPS fail in these settings, as the number of variational parameters must scale as $D \sim 2^{N}$. On the other hand, in a cavityQED quantum simulation multiple output fields are naturally accessible via polarization or higher order cavity modes, and at the same time large effective Hilbert space dimensions can be achieved, e.g., with trapped ions or atoms. With $N \gtrsim 10$, substantial practical speedups are already expected with respect to the classical CMPS algorithm, which requires a number of operations scaling as $2^{3 \times N}$.

The realization that ground-state CMPS and the field states emerging from a cavity are connected can be exploited to characterize the correlations of the emitted light. Indeed, we obtain a simple criteria to determine when the correlations in the light field are nonclassical: if it turns out that the simulated Hamiltonian is quadratic in the field operators; and (b) contains only "ultralocal" terms, i.e., no derivatives in the field operators, then the ground state is a trivial (i.e., Gaussian) product state, and there would be no nonclassical correlations in the emitted light.

The output of a cavity-QED apparatus admits a natural interpretation as a variational class of quantum-field states. We have demonstrated that this allows an analogue quantum simulation procedure for strongly correlated physics using current technology. This result opens up an entirely new perspective for all cavity-QED systems which exhibit sufficiently strong nonlinearities at the single-photon level. This includes not only optical cavities coupled to atoms, but also superconducting circuits with super-strong coupling to solid state quantum systems [58], as well as other nonlinear systems achieving a high optical depth without cavities, such as atomic ensembles exhibiting a Rydberg blockade [59], or coupled to nanophotonic waveguides $[60,61]$. Looking further afield, since the input-output and CMPS formalisms generalize in a natural way to 
fermionic settings [62-64], our simulation procedure might be applicable to cavity-like microelectronic settings involving fermionic degrees of freedom. We hope our work will inspire explorations of these promising directions.

We acknowledge helpful conversations and comments by Jens Eisert, Frank Verstraete, Ignacio Cirac, Bernhard Neukirchen, Jutho Haegeman, Konstantin Friebe, Jukka Kiukas, and Reinhard Werner. This work was supported by the Centre for Quantum Engineering and Space-Time Research (QUEST), the ERC Grant QFTCMPS, the Austrian Science Fund (FWF) through the SFB FoQuS (FWF Project No. F4003), the European Commission (AQUTE, COQUIT), the Engineering and Physical Sciences Research Council (EPSRC), and through the FET-Open Grant MALICIA (No. 265522).

*Deceased.

[1] R. P. Feynman, Int. J. Theor. Phys. 21, 467 (1982).

[2] S. Lloyd, Science 273, 1073 (1996).

[3] I. Buluta and F. Nori, Science 326, 108 (2009).

[4] A. Aspuru-Guzik and P. Walther, Nat. Phys. 8, 285 (2012).

[5] I. Bloch, J. Dalibard, and S. Nascimbène, Nat. Phys. 8, 267 (2012).

[6] M. Johanning, A. F. Varón, and C. Wunderlich, J. Phys. B 42, 154009 (2009).

[7] M. Lewenstein, A. Sanpera, V. Ahufinger, B. Damski, A. S. De, and U. Sen, Adv. Phys. 56, 243 (2007).

[8] A. Friedenauer, H. Schmitz, J. T. Glueckert, D. Porras, and T. Schaetz, Nat. Phys. 4, 757 (2008).

[9] R. Gerritsma, G. Kirchmair, F. Zähringer, E. Solano, R. Blatt, and C. F. Roos, Nature (London) 463, 68 (2010).

[10] E. Haller, R. Hart, M. J. Mark, J. G. Danzl, L. Reichsöllner, M. Gustavsson, M. Dalmonte, G. Pupillo, and H.-C. Nägerl, Nature (London) 466, 597 (2010).

[11] K. Kim, M.-S. Chang, S. Korenblit, R. Islam, E. E. Edwards, J. K. Freericks, G.-D. Lin, L.-M. Duan, and C. Monroe, Nature (London) 465, 590 (2010).

[12] B. P. Lanyon, J. D. Whitfield, G. G. Gillett, M. E. Goggin, M. P. Almeida, I. Kassal, J.D. Biamonte, M. Mohseni, B. J. Powell, M. Barbieri et al., Nat. Chem. 2, 106 (2010).

[13] R. Islam, E. E. Edwards, K. Kim, S. Korenblit, C. Noh, H. Carmichael, G.-D. Lin, L.-M. Duan, C.-C. J. Wang, J. K. Freericks et al., Nat. Commun. 2, 377 (2011).

[14] J. Simon, W. S. Bakr, R. Ma, M. E. Tai, P. M. Preiss, and M. Greiner, Nature (London) 472, 307 (2011).

[15] J. T. Barreiro, M. Müller, P. Schindler, D. Nigg, T. Monz, M. Chwalla, M. Hennrich, C.F. Roos, P. Zoller, and R. Blatt, Nature (London) 470, 486 (2011).

[16] B. P. Lanyon, C. Hempel, D. Nigg, M. Müller, R. Gerritsma, F. Zähringer, P. Schindler, J. T. Barreiro, M. Rambach, G. Kirchmair et al., Science 334, 57 (2011).

[17] L. Lepori, G. Mussardo, and A. Trombettoni, Europhys. Lett. 92, 50003 (2010).

[18] A. Bermudez, L. Mazza, M. Rizzi, N. Goldman, M. Lewenstein, and M. A. Martin-Delgado, Phys. Rev. Lett. 105, 190404 (2010).
[19] J. I. Cirac, P. Maraner, and J. K. Pachos, Phys. Rev. Lett. 105, 190403 (2010).

[20] F. L. Semião and M. Paternostro, Quantum Inf. Process. 11, 67 (2012).

[21] E. Kapit and E. Mueller, Phys. Rev. A 83, 033625 (2011).

[22] M. J. Hartmann, F. G. S. L. Brandão, and M. B. Plenio, Nat. Phys. 2, 849 (2006).

[23] A. D. Greentree, C. Tahan, J.H. Cole, and L.C. L. Hollenberg, Nat. Phys. 2, 856 (2006).

[24] D. G. Angelakis, M. F. Santos, and S. Bose, Phys. Rev. A 76, 031805 (2007).

[25] T. Byrnes and Y. Yamamoto, Phys. Rev. A 73, 022328 (2006).

[26] K. Temme, T. J. Osborne, K. G. Vollbrecht, D. Poulin, and F. Verstraete, Nature (London) 471, 87 (2011).

[27] S. P. Jordan, K. S. M. Lee, and J. Preskill, Science 336, 1130 (2012).

[28] S.P. Jordan, K.S.M. Lee, and J. Preskill, arXiv: $1112.4833 \mathrm{v} 1$

[29] J. M. Raimond, M. Brune, and S. Haroche, Rev. Mod. Phys. 73, 565 (2001).

[30] R. Miller, T. E. Northup, K. M. Birnbaum, A. Boca, A. D. Boozer, and H. J. Kimble, J. Phys. B 38, S551 (2005).

[31] H. Walther, B. T. H. Varcoe, B.-G. Englert, and T. Becker, Rep. Prog. Phys. 69, 1325 (2006).

[32] S. Haroche and J.M. Raimond, Exploring the Quantum: Atoms, Cavities, and Photons (Oxford University Press, New York, 2006).

[33] S. M. Girvin, M. H. Devoret, and R. J. Schoelkopf, Phys. Scr. T137, 014012 (2009).

[34] We define $\hat{E}^{+}(t)$ in units such that $\left\langle\hat{E}^{-}(t) \hat{E}^{+}(t)\right\rangle$ corresponds to the mean number of photons exiting the cavity per unit time.

[35] R. J. Glauber, Phys. Rev. 130, 2529 (1963).

[36] L. Mandel and E. Wolf, Optical Coherence and Quantum Optics (Cambridge University Press, New York, 1995), cf. in particular Chap. 12

[37] S. Gerber, D. Rotter, L. Slodicka, J. Eschner, H. J. Carmichael, and R. Blatt, Phys. Rev. Lett. 102, 183601 (2009).

[38] M. Koch, C. Sames, M. Balbach, H. Chibani, A. Kubanek, K. Murr, T. Wilk, and G. Rempe, Phys. Rev. Lett. 107, 023601 (2011).

[39] See Supplemental Material at http://link.aps.org/ supplemental/10.1103/PhysRevLett.110.090501 for the identification of the output field of a cavity QED system and continuous matrix product states, and further details on the quantum variational algorithm.

[40] F. Verstraete and J. I. Cirac, Phys. Rev. Lett. 104, 190405 (2010).

[41] T. J. Osborne, J. Eisert, and F. Verstraete, Phys. Rev. Lett. 105, 260401 (2010).

[42] J. Haegeman, J. I. Cirac, T. J. Osborne, H. Verschelde, and F. Verstraete, Phys. Rev. Lett. 105, 251601 (2010).

[43] J. Haegeman, J. I. Cirac, T. J. Osborne, H. Verschelde, and F. Verstraete, Proc. Sci., FacesQCD2010 (2010) 029.

[44] M. Fannes, B. Nachtergaele, and R. F. Werner, Commun. Math. Phys. 144, 443 (1992).

[45] F. Verstraete, V. Murg, and J. I. Cirac, Adv. Phys. 57, 143 (2008).

[46] J. I. Cirac and F. Verstraete, J. Phys. A 42, 504004 (2009). 
[47] U. Schollwöck, Ann. Phys. (Amsterdam) 326, 96 (2011).

[48] S. R. White, Phys. Rev. Lett. 69, 2863 (1992).

[49] U. Schollwöck, Rev. Mod. Phys. 77, 259 (2005).

[50] D. Jennings and T. J. Osborne (unpublished).

[51] M. J. Collett and C. W. Gardiner, Phys. Rev. A 30, 1386 (1984).

[52] C. Schön, E. Solano, F. Verstraete, J. I. Cirac, and M. M. Wolf, Phys. Rev. Lett. 95, 110503 (2005).

[53] C. Schön, K. Hammerer, M. M. Wolf, J. I. Cirac, and E. Solano, Phys. Rev. A 75, 032311 (2007).

[54] E.H. Lieb and W. Liniger, Phys. Rev. 130, 1605 (1963).

[55] F. Dubin, C. Russo, H. G. Barros, A. Stute, C. Becher, P. O. Schmidt, and R. Blatt, Nat. Phys. 6, 350 (2010).

[56] A. Stute, B. Casabone, P. Schindler, T. Monz, P. O. Schmidt, B. Brandstätter, T.E. Northup, and R. Blatt, Nature (London) 485, 482 (2012).
[57] P. Hauke, F. M. Cucchietti, L. Tagliacozzo, I. Deutsch, and M. Lewenstein, Rep. Prog. Phys. 75, 082401 (2012).

[58] D. Bozyigit, C. Lang, L. Steffen, J. M. Fink, C. Eichler, M. Baur, R. Bianchetti, P. J. Leek, S. Filipp, M. P. Da Silva et al., Nat. Phys. 7, 154 (2010).

[59] J.D. Pritchard, K. J. Weatherill, and C. S. Adams, in Annual Review of Cold Atoms and Molecules, edited by K. Madison et al. (World Scientific, Singapore, 2013), Vol. 1, pp. 301-350.

[60] E. Vetsch, D. Reitz, G. Sagué, R. Schmidt, S. T. Dawkins, and A. Rauschenbeutel, Phys. Rev. Lett. 104, 203603 (2010).

[61] A. Goban, K. S. Choi, D. J. Alton, D. Ding, C. Lacroûte, M. Pototschnig, T. Thiele, N. P. Stern, and H. J. Kimble, Phys. Rev. Lett. 109, 033603 (2012).

[62] H. B. Sun and G. J. Milburn, Phys. Rev. B 59, 10748 (1999).

[63] C. P. Search, S. Pötting, W. Zhang, and P. Meystre, Phys. Rev. A 66, 043616 (2002).

[64] C. W. Gardiner, Opt. Commun. 243, 57 (2004). 